\title{
Reliability of Vibrating Mesh Technology
}

\author{
Ashwin A Gowda DO, Ann D Cuccia MPH RRT-NPS RPFT, and Gerald C Smaldone MD PhD
}

\begin{abstract}
BACKGROUND: For delivery of inhaled aerosols, vibrating mesh systems are more efficient than jet nebulizers are and do not require added gas flow. We assessed the reliability of a vibrating mesh nebulizer (Aerogen Solo, Aerogen Ltd, Galway Ireland) suitable for use in mechanical ventilation. METHODS: An initial observational study was performed with 6 nebulizers to determine run time and efficiency using normal saline and distilled water. Nebulizers were run until cessation of aerosol production was noted, with residual volume and run time recorded. Three controllers were used to assess the impact of the controller on nebulizer function. Following the observational study, a more detailed experimental protocol was performed using 20 nebulizers. For this analysis, 2 controllers were used, and time to cessation of aerosol production was noted. Gravimetric techniques were used to measure residual volume. Total nebulization time and residual volume were recorded. Failure was defined as premature cessation of aerosol production represented by residual volume of $>10 \%$ of the nebulizer charge. RESULTS: In the initial observational protocol, an unexpected sporadic failure rate was noted of $25 \%$ in 55 experimental runs. In the experimental protocol, a failure rate was noted of $30 \%$ in 40 experimental runs. Failed runs in the experimental protocol exhibited a wide range of retained volume averaging \pm SD $36 \pm 21.3 \%$ compared with $3.2 \pm 1.5 \%(P=.001)$ in successful runs. Small but significant differences existed in nebulization time between controllers. CONCLUSIONS: Aerogen Solo nebulization was often randomly interrupted with a wide range of retained volumes. Key words: nebulizer; mechanical ventilation; aerosol; efficiency; residual volume; run time. [Respir Care 2017;62(1):65-69. (C) 2017 Daedalus Enterprises]
\end{abstract}

\section{Introduction}

Our group is interested in the delivery of inhaled drugs to intubated mechanically ventilated patients. ${ }^{1-3}$ Treatment algorithms require a device that is efficient and reliable

\footnotetext{
Drs Gowda and Smaldone are affiliated with the Division of Pulmonary, Critical Care and Sleep Medicine, Department of Medicine, State University of New York at Stony Brook, Stony Brook, New York; Ms Cuccia is affiliated with the Respiratory Care Program, School of Health, Technology and Management, State University of New York at Stony Brook, Stony Brook, New York. Departmental funds were used to support this study. The authors have disclosed no conflicts of interest.

Supplementary material related to this paper is available at http:// www.rcjournal.com.

Correspondence: Ashwin Gowda, DO, Division of Pulmonary, Critical Care and Sleep Medicine, Department of Medicine, State University of New York at Stony Brook, T17-040 Health Sciences Center, Stony Brook, NY 11794. E-mail: ashwin.gowda@stonybrook.edu.
}

DOI: $10.4187 /$ respcare.04702 with repeated use. Historically, jet nebulizers have been the standard delivery system for aerosolized medications. ${ }^{4}$ They suffer from being relatively inefficient and require an external air source to operate. Further, there are no regulatory standards for nebulizer delivery during mechanical ventilation, resulting in limited control of the dose to the patient. ${ }^{5}$ For patients receiving bronchodilator therapy, the treatment effect is quickly clinically apparent, and the dose can be titrated at the bedside. Bronchodilators are potent drugs (micrograms delivered to the patient) and safe with relatively flat dose-response curves. ${ }^{3}$ For less potent agents such as antibiotics (milligrams needed), clinical effects are dependent on drug levels, and unlike bronchodilators, response is not apparent when simply observing the patient. To maximize the success of a clinical trial and ensure adequate control of dosage, external controls of aerosol delivery may be required, such as the type of ventilator, control of breathing patterns, breath actuation, and humidification. Aerosol output can be measured directly, for example, by using a radioactive tracer or drug assay. Devices can also be screened using gravimetric techniques before embarking on more de- 
tailed studies. ${ }^{6}$ Failure to pretest delivery systems for function and efficiency often leads to failure of a clinical trial. ${ }^{7,8}$

An additional factor affecting reproducibility of drug delivery is inter- and intra-device variability. That is, in a clinical trial does each example of the device deliver the same aerosol with repeated use? Is the technology robust (intra-device variability)? In published studies of jet nebulizer technology, pretesting of devices before clinical trials has revealed that jet nebulizers can be robust, performing reproducibly over time in terms of both drug output and particle distribution. ${ }^{9-11}$ However, jet nebulizers have significant inter-device variability. A comparison of 23 nebulizer/compressor combinations showed that particle distribution and output varied greatly between devices. ${ }^{12}$ This gives further evidence that rigorous testing of a nebulizer should be done before any pharmacologic study is conducted so that baseline characteristics are understood.

Vibrating mesh technology is an alternative to jet nebulization. Mesh devices are reported to be more efficient than jet nebulizers are and do not contribute added gas to the ventilator circuit. On the other hand, compared with jet nebulizers, vibrating mesh devices can be more sensitive to contaminant, ${ }^{13}$ and device orientation ${ }^{14}$ and have sensitive electronic controls. Before defining a human study protocol, we decided to test available mesh technology for reliability in delivering a repeated dose. To our knowledge the Aeroneb Solo (Aerogen, Galway, Ireland) is currently the only stand-alone, single-patient mesh device commercially available for use in mechanically ventilated patients. Previous studies have reported delivery efficiency $(54.5 \pm 0.4 \%)$ and particle distributions (mass median aerodynamic diameter $4.6 \pm 0.5 \mu \mathrm{m}$ to $5.9 \pm 5.1 \mu \mathrm{m}$ ) for the Solo. ${ }^{16-21}$ However, those reports tested limited examples of the device with limited numbers of experimental runs. The Aerogen Solo has also been reported by the manufacturer to have residual volumes of $<0.1 \mathrm{~mL}$ with normal use (http://www.aerogen.com, Accessed February 25,2016 ). Our group has tested other forms of mesh technology and found significant variability in device function compared with the function of jet nebulizers. ${ }^{14}$ In addition, a recent report demonstrated variability from different manufacturers using the same dose of tobramycin. ${ }^{15}$ It therefore remains uncertain whether mesh technology provides reproducible drug delivery for repeated use in a typical clinical trial. The primary objective of this study was to determine the reliability of the Aerogen Solo vibrating mesh nebulizer to repeatedly deliver the expected dose of a test aerosol.

\section{Methods}

\section{Observational Protocol}

First, we designed an observational study to determine the function of new devices to assess possible study pa-

\section{QUICK LOOK}

\section{Current knowledge}

Standards regulating the function of nebulizers are limited. Vibrating mesh devices are known to be convenient and efficient, but limited data are available regarding reliability with repeated use.

\section{What this paper contributes to our knowledge}

The Aerogen Solo, the only single-patient use mesh device available for use during mechanical ventilation, was efficient but frequently failed to deliver the expected dose.

rameters to be used in a more intensive follow-up study. In the initial observational study, 6 nebulizers were tested along with different controllers, solutions, and fill volumes (see supplemental file at http://www.rcjournal.com). To standardize our test runs, the devices were suspended via a ring stand at the angle and direction specified by the manufacturer and visually observed. Different volumes of test formulations (normal saline or distilled water) were used. The aerosol cloud was readily apparent, and the duration of nebulization was recorded (run time, min:s) at the time aerosol production ceased. In addition, visual residual volume, if any, was noted. Our first assumption was that each device would empty completely and repeatedly (a measure of reproducible high efficiency). Failure to empty would result in the visual observation of a residual volume, which was quantified by extracting the liquid with a syringe. We initially tested 3 nebulizer controllers, an older version used in studies of other Aerogen prototypes (Controller "A," Aerogen PRO) and 2 new controllers specified for use with the Solo (Controllers "B" and "C," Aerogen PRO-X). A functions check of the power cord and controller was performed before the start of nebulization, and the controller was operated in accordance with manufacturer's instructions for normal operation.

\section{Experimental Study and Protocol}

In the observational study, the nebulizers were found to have significant inter- and intra-device variability. We decided to perform a more detailed experimental study to further quantify the variables defining device reliability. The test solution was fixed at $3 \mathrm{~mL}$ of normal saline, a suitable surrogate for multiple water-soluble drugs. ${ }^{3,11,22,23}$ Twenty nebulizers were studied, including 4 from the observational protocol and 4 previously used nebulizers from our own laboratory supply. Twelve additional new nebulizers were added to the test protocol. The protocol utilized 2 new Aerogen Pro-X controllers (Controllers B and C). 

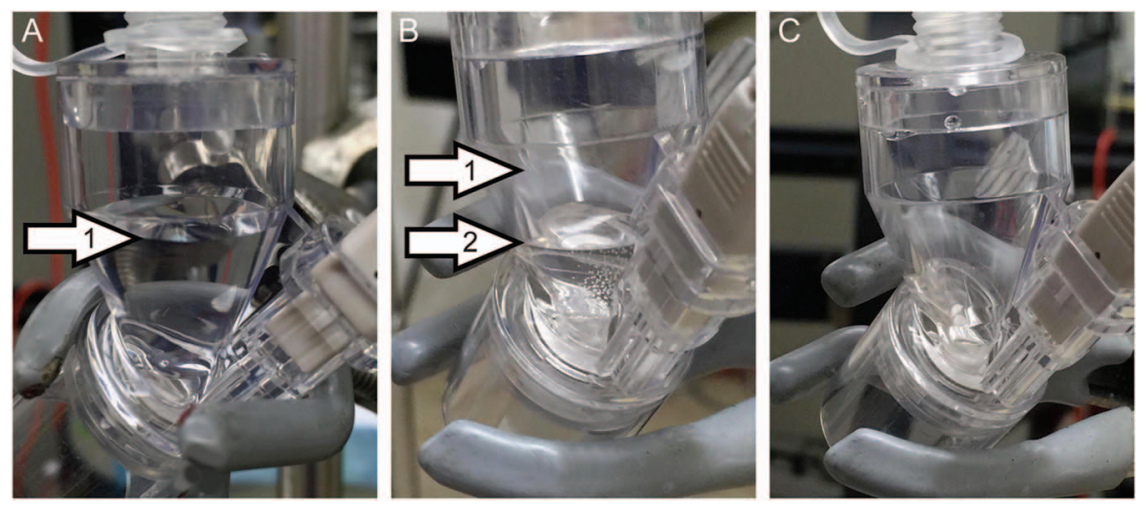

Fig. 1. Example of Aerogen Solo Nebulizer before use with a fill volume of $3 \mathrm{~mL}$ normal saline (arrow 1) (A); the same nebulizer after random premature cessation of nebulization, with residual volume of $0.4 \mathrm{~mL}$ (arrow 2) (B); the same nebulizer after a successful run with minimal residual volume $(C)$.

We did not include Controller A used in the observational protocol, because it is not currently commercially available. To compare nebulization times for each controller, we examined devices that had successful runs (no premature cessation of aerosol production) with both controllers (B and C). A statistical comparison was performed using the Wilcoxon matched-pairs signed rank test, (GraphPad Prism, GraphPad Software, San Diego, California). Change in weight was used to define nebulizer output. At the first cessation of nebulization, the nebulizer was moved from the ring stand and weighed (Mettler-Toledo AT 261 Delta Range Scale, Columbus, Ohio). Failure was defined as having $>10 \%$ residual volume after cessation of nebulization or a pre- and postnebulization weight difference of $<2.7 \mathrm{~g}$. Percentage of the retained nebulizer charge (\% residual volume) at the cessation of nebulization was also analyzed for both successful and failed runs using the Mann-Whitney, nonparametric test (GraphPad Prism, GraphPad Software, San Diego, California).

\section{Results}

Supplementary Table 1 shows the results of the initial observational protocol (see the supplementary table at http://www.rcjournal.com). It includes total time of nebulization for each device, controller used, and residual volume remaining if nebulization prematurely terminated. All 6 of the devices tested in the observational protocol had sporadic failures during experimental runs. For example, Figure 1A shows an Aerogen Solo nebulizer before use with a $3-\mathrm{mL}$ fill volume of normal saline. Figure $1 \mathrm{~B}$ shows visible residual volume $(0.4 \mathrm{~mL})$ after premature cessation of nebulization, and Figure 1C shows the same nebulizer after another run where it emptied as expected with minimal residual volume. Using our criteria for device failure, we found that premature cessation of nebulization occurred, with significant residual volume on 14/55 (25\%) of ex-
Table 1. Controller Times

\begin{tabular}{|c|c|c|c|c|}
\hline \multirow{2}{*}{ Study } & \multirow{2}{*}{ Controller } & \multirow{2}{*}{$\begin{array}{l}\text { Runs } \\
\text { (no.) }\end{array}$} & Time $(\min )$ & \multirow{2}{*}{$P$} \\
\hline & & & Mean \pm SD & \\
\hline \multirow[t]{2}{*}{ Experimental } & B & 20 & $11.3 \pm 2.7$ & $.001 *$ \\
\hline & $\mathrm{C}$ & 20 & $9.6 \pm 2.4$ & \\
\hline
\end{tabular}

perimental runs. Total time (min:s) to cessation of nebulization appeared to vary widely (5:11 to $18: 51)$ as did residual volume $(0.2 \mathrm{~mL}$ to $1.2 \mathrm{~mL})$. Premature cessation of nebulization did not appear related to the controller used.

Tables 1 and 2 show the results of data obtained from the experimental protocol. Failure to empty was noted on $12 / 40(30 \%)$ of experimental runs. Devices demonstrated random failure with significant variability in the retained residual volume (failure mean $\pm S D$ residual volume $36 \pm 21.3 \%$ ) compared with runs nebulized to completion $(3.2 \pm 1.5 \%)(P=.001)$. Small but significant differences were noted in total time of nebulization, depending on the controller used (Controller B [11.3 $\pm 2.7 \mathrm{~min}]$ vs C [9.6 $\pm 2.4 \mathrm{~min}, P=.001])$.

\section{Discussion}

The objective of this study was to assess the reliability of the Aerogen Solo with repeated use. Our findings indicate that the nebulizer will often randomly stop nebulizing. For a $3.0-\mathrm{mL}$ dose, the manufacturer has stated that residual volumes with normal use should be on average $<0.1 \mathrm{~mL}$. Therefore, we expected each device to empty nearly completely in our test protocols. Failed devices often restarted if moved or behaved differently in subsequent experiments. In addition, the residual volumes 
Table 2. Experimental Protocol: Residual Volumes

\begin{tabular}{|c|c|c|c|c|}
\hline \multirow{2}{*}{ Neb. } & \multirow{2}{*}{ Controller } & \multirow{2}{*}{ Run No. } & \multicolumn{2}{|c|}{ Residual Volume (\%) } \\
\hline & & & $\leq 10 \%$ (28 runs) & $>10 \%$ (12 runs) \\
\hline 3 & $\mathrm{~B}$ & 17 & 2.3 & \\
\hline 3 & $\mathrm{C}$ & 18 & 2.9 & \\
\hline 4 & $\mathrm{~B}$ & 7 & 2.8 & \\
\hline 4 & $\mathrm{C}$ & 8 & 3.4 & \\
\hline 5 & $\mathrm{~B}$ & 6 & 3.1 & \\
\hline 5 & $\mathrm{C}$ & 7 & 3.5 & \\
\hline 6 & B & 3 & 2.7 & \\
\hline 6 & $\mathrm{C}$ & 4 & 2.0 & \\
\hline 7 & B & 3 & & 21.1 \\
\hline 7 & $\mathrm{C}$ & 4 & & 35.3 \\
\hline 8 & B & 3 & 2.1 & \\
\hline 8 & $\mathrm{C}$ & 4 & 4.7 & \\
\hline 9 & $\mathrm{~B}$ & 3 & & 84.6 \\
\hline 9 & $\mathrm{C}$ & 4 & & 49.6 \\
\hline 10 & $\mathrm{~B}$ & 3 & 3.9 & \\
\hline 10 & $\mathrm{C}$ & 4 & 8.8 & \\
\hline 11 & $\mathrm{C}$ & 1 & & 18.2 \\
\hline 11 & $\mathrm{~B}$ & 2 & 3.1 & \\
\hline 12 & B & 1 & 5.1 & \\
\hline 12 & $\mathrm{C}$ & 2 & & 31.8 \\
\hline 13 & $\mathrm{~B}$ & 2 & 1.8 & \\
\hline 13 & $\mathrm{C}$ & 1 & 3.9 & \\
\hline 14 & $\mathrm{~B}$ & 1 & 2.2 & \\
\hline 14 & $\mathrm{C}$ & 2 & 1.8 & \\
\hline 15 & B & 2 & 3.7 & \\
\hline 15 & $\mathrm{C}$ & 1 & 1.7 & \\
\hline 16 & B & 1 & 0.4 & \\
\hline 16 & $\mathrm{C}$ & 2 & 4.8 & \\
\hline 17 & B & 2 & 2.3 & \\
\hline 17 & $\mathrm{C}$ & 1 & 2.8 & \\
\hline 18 & B & 2 & & 21.9 \\
\hline 18 & $\mathrm{C}$ & 1 & & 56.5 \\
\hline 19 & B & 1 & 3.8 & \\
\hline 19 & $\mathrm{C}$ & 2 & & 53.6 \\
\hline 20 & B & 2 & 3.4 & \\
\hline 20 & $\mathrm{C}$ & 1 & & 11.8 \\
\hline 21 & B & 1 & & 30.3 \\
\hline 21 & $\mathrm{C}$ & 2 & & 17.6 \\
\hline 22 & B & 2 & 4.5 & \\
\hline \multirow[t]{4}{*}{22} & $\mathrm{C}$ & 1 & 2.2 & \\
\hline & & Mean & 3.2 & 36.0 \\
\hline & & SD & 1.5 & 21.3 \\
\hline & & $P *$ & \multicolumn{2}{|c|}{$<.001$} \\
\hline
\end{tabular}

* Mann-Whitney rank-sum test.

varied widely from run to run, indicating significant interand intra-device variability. Table 1 indicates that differences between controllers although significant were small. Residual volumes for the completed runs were consistent with those reported by the manufacturer. These observations suggest that failure was not in the electronics or due to the mesh but indicated a problem with the gravitational feed. Currently marketed mesh devices rely on gravity to maintain contact between the liquid column and the vibrating mesh. In designing our protocol, we accepted a more liberal residual volume $(0.3 \mathrm{~mL}, 3$ times the expected) but much less than the usual residual volume for a jet nebulizer. This is equivalent to $10 \%$ residual of the initial nebulizer charge (eg, the expected weight change for a 3-mL fill volume should be at least $2.7 \mathrm{~g}$ ) between the pre- and poststoppage weights ( $2.7 \mathrm{~g}$ being equivalent to $2.7 \mathrm{~mL}$ of normal saline). As opposed to other nebulizer technologies, the Aerogen device expresses significant random variability. Direct numeric comparisons with other devices are therefore difficult.

Gravimetric analysis has been previously used as a surrogate method for assessing nebulizer output. For jet nebulizers, it has been reported to overestimate aerosol production. ${ }^{6}$ However, the gravimetric method was sufficient to demonstrate variability in nebulizer output. Had the device functioned reproducibly, then more direct measurements of aerosol output and particle size would have been possible.

The available literature suggests that the mesh nebulizer is a reliable, efficient delivery device suitable for a ventilator circuit. In addition, there are no competing nebulizer devices for drug delivery that are formally marketed for ventilator use. Therefore, in recent years, mesh technology has become commonly accepted for use with mechanical ventilation. In our hands, older versions of this technology were more reliable but, according to the manufacturer, they are no longer supported. We have a need for a wellcharacterized reliable delivery system for drug delivery, and, if selected, a given device must be commercially available, hence our study. Our in vitro laboratory-testing algorithm commonly includes repetitive measurement of drug delivery and particle distribution. The present study, however, was limited, because the tested devices would not reliably empty, and we could not perform consistent output studies; therefore, we cannot comment on overall device efficiency or particle delivery.

What does our study mean to the practicing clinician? In general, clinicians should be aware that, at present, no nebulizer/drug combinations are cleared for use with mechanical ventilation, and it is the clinician's responsibility to ensure adequate delivery. Our study reveals the possibility that the Aerogen device may abruptly stop before all drug has been delivered. With this information, clinicians should monitor patients during treatment for interruption of nebulization to minimize variability of dosing. The present study was not designed to compare different devices or technologies. However, as summarized in the introduction, many studies have been published that report drug delivery using jet nebulization and, for bronchodilators, the metered-dose inhaler. ${ }^{24}$ These devices are, in general, re- 


\section{Reliability of Vibrating Mesh Nebulizer}

liable, but they vary markedly in efficiency and particle distribution, and successful investigator-driven clinical trials require that the devices be well characterized beforehand.

\section{Conclusions}

Our aim was to assess the Aerogen Solo for inter- and intra-device reliability. We found that nebulization was often randomly interrupted with a wide range of retained volumes.

\section{ACKNOWLedgMenT}

We thank Lorraine Morra for assistance with data analysis and preparation of the manuscript.

\section{ReFERENCES}

1. Palmer LB, Smaldone GC. Reduction of bacterial resistance with inhaled antibiotics in the intensive care unit. Am J Respir Crit Care Med 2014;189(10):1225-1233.

2. Palmer LB, Smaldone GC, Simon SR, O'Riordan TG, Cuccia A. Aerosolized antibiotics in mechanically ventilated patients: delivery and response. Crit Care Med 1998;26(1):31-39.

3. Miller DD, Amin MM, Palmer LB, Shah AR, Smaldone GC. Aerosol delivery and modern mechanical ventilation: in vitro/in vivo evaluation. Am J Respir Crit Care Med 2003;168(10):1205-1209.

4. Dolovich MB, Ahrens RC, Hess DR, Anderson P, Dhand R, Rau JL, et al. Device selection and outcomes of aerosol therapy: Evidencebased guidelines: American College of Chest Physicians/American College of Asthma, Allergy, and Immunology. Chest 2005;127(1): 335-371.

5. McPeck M, O'Riordan TG, Smaldone GC. Choice of mechanical ventilator: Influence on nebulizer performance. Respir Care 1993; 38:887-895.

6. Tandon R, McPeck M, Smaldone GC. Measuring nebulizer output. Aerosol production vs gravimetric analysis. Chest 1997;111(5):13611365.

7. Kofteridis DP, Alexopoulou C, Valachis A, Maraki S, Dimopoulou D, Georgopoulos D, Samonis G. Aerosolized plus intravenous colistin versus intravenous colistin alone for the treatment of ventilatorassociated pneumonia: a matched case-control study. Clin Infect Dis 2010;51(11):1238-1244.

8. Rattanaumpawan P, Lorsutthitham J, Ungprasert P, Angkasekwinai $\mathrm{N}$, Thamlikitkul V. Randomized controlled trial of nebulized colistimethate sodium as adjunctive therapy of ventilator-associated pneumonia caused by gram-negative bacteria. J Antimicrob Chemother 2010;65(12):2645-2649.

9. Cabahug CJ, McPeck M, Palmer LB, Cuccia A, Atkins HL, Smaldone GC. Utility of technetium-99m-DTPA in determining regional ventilation. J Nucl Med 1996;37(2):239-244.
10. Smaldone GC, Cruz-Rivera M, Nikander K. In vitro determination of inhaled mass and particle distribution for budesonide nebulizing suspension. J Aerosol Med 1998;11:113-125.

11. Smaldone GC, Fuhrer J, Steigbigel RT, McPeck M. Factors determining pulmonary deposition of aerosolized pentamidine in patients with human immunodeficiency virus infection. Am Rev Respir Dis 1991;143(4 Pt 1):727-737.

12. Smith EC, Denyer J, Kendrick AH. Comparison of twenty-three nebulizer/compressor combinations for domiciliary use. Eur Respir J 1995;8(7):1214-1221.

13. Rottier BL, van Erp CJ, Sluyter TS, Heijerman HG, Frijlink HW, Boer AH. Changes in performance of the Pari eFlow rapid and Pari LC Plus during 6 months use by CF patients. J Aerosol Med Pulm Drug Deliv 2009;22(3):263-269.

14. Skaria S, Smaldone GC. Omron NE. U22: Comparison between vibrating mesh and jet nebulizer. J Aerosol Med Pulm Drug Deliv 2010;23(3):173-180.

15. Mashat M, Clark BJ, Assi KH, Chystyn H. In vitro aerodynamic characterization of the dose emitted during nebulization of tobramycin high strength solution by novel and jet nebulizer delivery systems. Pulm Pharmacol Ther Epub 2015 Dec 30.

16. Sidler-Moix AL, Di Paolo ER, Dolci U, Berger-Gryllaki M, Cotting J, Pannatier A. Physicochemical aspects and efficiency of albuterol nebulization: comparison of three aerosol types in an in vitro pediatric model. Respir Care 2015;60(1):38-46.

17. Alhamad BR, Fink JB, Harwood RJ, Sheard MM, Ari A. Effect of aerosol devices and administration techniques on drug delivery in a simulated spontaneously breathing pediatric tracheostomy model. Respir Care 2015;60(7):1026-1032.

18. Ari A, de Andrade AD, Sheard M, AlHamad B, Fink JB. Performance comparisons of jet and mesh nebulizers using different interfaces in simulated spontaneously breathing adults and children. J Aerosol Med Pulm Drug Deliv 2015;28(4):281-289.

19. Ari A, Atalay OT, Harwood R, Sheard MM, Aljamhan EA, Fink JB. Influence of nebulizer type, position, and bias flow on aerosol drug delivery in simulated pediatric and adult lung models during mechanical ventilation. Respir Care 2010;55(7):845-851.

20. Davidson WJ, Dennis J, The S, Litoski B, Pieron C, Leigh R. Identification and validation of nebulized aerosol devices for sputum induction. Can Respir J 2014;21(2):101-106.

21. Michotte JB, Jossen E, Roeseler J, Liistro G, Reychler G. In vitro comparison of five nebulizers during noninvasive ventilation: analysis of inhaled and lost doses. J Aerosol Med Pulm Drug Deliv 2014;27(6):430-440.

22. Diaz KT, Skaria S, Harris K, Solomita M, Lau S, Bauer K, et al. Delivery and safety of inhaled interferon-gamma in idiopathic pulmonary fibrosis. J Aerosol Med Pulm Drug Deliv 2012;25(2):79-87.

23. McPeck M, Tandon R, Hughes K, Smaldone GC. Aerosol delivery during continuous nebulization. Chest 1997;111(5):1200-1205.

24. Diot P, Morra L, Smaldone GC. Albuterol delivery in a model of mechanical ventilation. Comparison of metered-dose inhaler and nebulizer efficiency. Am J Respir Crit Care Med 1995;152(4 Pt 1):13911394. 